\title{
Analysis of Grey-Level Features for Line Segment Stereo Matching
}

\author{
Oliver Schreer, Irmfried Hartmann and Roger Adams \\ Institute for Measurement and Automation Technology \\ Department of Electrical Engineering, Technical University Berlin \\ E-mail: schreer@rtws18.ee.TU-Berlin.DE
}

\begin{abstract}
In order to recover 3D-information from stereo image pairs, a number of stereo matching methods are available. These methods mainly differ in various tokens which are applied to solve the correspondence problem between left and right image. In most of the line segment based stereo matching algorithms, geometrical and structural information is used to estimate the correspondence. Unfortunately these features are dependent on the accuracy of segmentation and the angle of view. This might cause an ambiguity regarding the solution. Grey-level features (GLF) are introduced as robust features independent of segmentation errors and the angle of view. Concerning different line segmentation algorithms, e.g. sequential-oriented or global-oriented algorithms, two methods are proposed for a proper estimation of grey-level information. Finally this additional information will be applied in a line segment based stereo matching algorithm. Regarding these different grey-level features the uniqueness of the solution and the computational effort will be compared with geometrical features. Experimental results are presented.
\end{abstract}

\section{Introduction}

Many vision based applications, e.g. navigation of autonomous robots, involve recovering $3 \mathrm{D}$-information from $2 \mathrm{D}$-images. In order to solve this problem, two different images of the same scene are needed, and the correspondence between different tokens in two images [2] is to establish. There is a wide range of tokens, e.g. simple points, straight line segments and more complex image structures. The matching strategy is definitely depending on the type of token respectively their combination. Straight line segments are suitable tokens for the description of industrial or manmade environment. The most important constraint for stereo matching is the epipolar constraint, but for an unambigious match additional constraints like geometrical, structural and contrast constraints are needed due to parallel line segments or segmentation errors [6] [7] [8]. The next chapter defines grey-level features, based on the original image, which extend the contrast constraint. Different strategies are proposed to estimate them depending on the segmentation algorithm. In chapter 3 , this additional features are applied in a line segment based stereo matching algorithm [1], and their reliability and complexity will be discussed.

\section{Estimation of Grey-Level Features (GLF)}

Geometrical and structural features are unfortunately sensitive to segmentation errors and depending on the angle of view, which results in an ambiguous solution. Considering the grey-level image and the intensity change regarding line segments, the following three features become obvious: 
- the intensity difference at the edge, the gradient

- the direction of intensity change, the sign of the gradient as a binary feature

- the mean value across the edge

The advantages of these features are their simplicity, robustness against segmentation errors and view-point independency.

First of all, the edge image has to be calculated with an operator, which should show the following characteristics: a one-pixel-width edge image, correct placement and robustness against noise. In the experiments a simplified edge operator based on Haralick's facet model [5] is used, which meets these requirements. Furthermore, line segments will be extracted from edge images, to which sequential [9] or more global segmentation algorithms [4] like Hough transform can be applied.

\subsection{Direct Feature Estimation in Sequential-Oriented Segmentation Algorithms}

Concerning sequential-oriented algorithms, the grey-level features could be estimated during the segmentation process. The gradient (Grad) of a segment is defined as the mean value of the gradient of all edge points which are part of the segment.

$$
\begin{aligned}
& \operatorname{grad}_{e}(i): \text { Gradient value of an edge point } \\
& \operatorname{Grad}_{\text {seg }}=\frac{1}{n} \sum_{i=1}^{n} \operatorname{grad}_{e}(i), \quad n \text { : number of edge points }
\end{aligned}
$$

This gradient definition depends on the single gradient values of each edge point determined by the the type of edge detector. Regarding this fact, the accuracy of estimation depends on the used method. The direction of intensity change $(D I C)$ is the sign of the gradient. It is also determined by the applied algorithm.

$$
D I C_{\text {seg }}=\operatorname{sign}\left(\operatorname{Grad}_{\text {seg }}\right) \text {, }
$$

The mean grey value $(M G V)$ has to be estimated as the mean sum of intensity values in a defined neighbourhood around the edge points of the segment.

$$
\begin{aligned}
& I_{e}(i, j): \text { Intensity value of an edge point in a defined neighbourhood } \\
& M G V_{\text {seg }}=\frac{1}{n m} \sum_{i=1}^{n} \sum_{j=1}^{m} I_{e}(i, j) \quad \begin{array}{l}
n: \text { number of edge points } \\
m: \text { number of neighbours }
\end{array}
\end{aligned}
$$

In the figure below an example is given of edge points corresponding to an edge. The neighbourhood is determined by a mask of size $=5$. The distance between the centers of masks has to be the size of the mask, guaranteeing no overlap of the masks, i.e. no double summation of pixels.

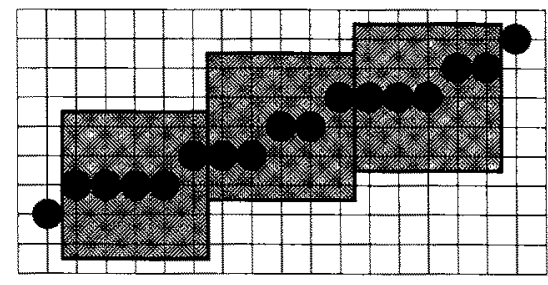

Fig.1. Example of neighbourhood definition 


\subsection{Feature Estimation in Global-Oriented Segmentation Algorithms}

Regarding the feature estimation from a more global point of view, it is obvious to define the gradient at an edge as the difference between the intensity of each side of the edge. The definition of each side is determined by the orientation and slope $m$ of the segment, given by the line segment parameters, e.g. $x / y$-coordinates of the end points or start point, angle and length of the segment.

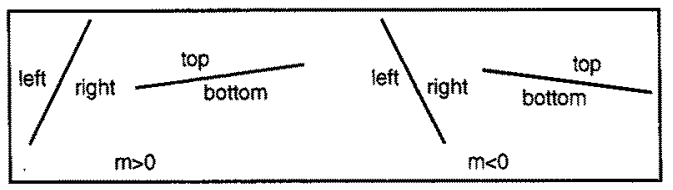

Fig.2. Definition of segment sides

$I_{l / r / t / b}$ : Mean Intensity with $l:$ left, $r$ :right, $t:$ top, $b$ : bottom

$\operatorname{Grad}_{\text {seg }}=I_{l / t}-I_{r / b}$,

The direction of intensity change is the sign of the gradient again, and the mean grey value is the mean sum of mean intensities.

$$
M G V_{s e g}=\left(I_{l / t}+I_{r / b}\right) / 2,
$$

What do we understand by mean intensity? Considering a real edge, approached with a line segment, there are two regions on the leftright or top/bottom side, each of them with approximately the same intensity values. These regions are limited in straight line direction by a cut-off parameter, called border. A second parameter, the neighbourhood is limiting the region parallel to the segment. Fig. 3 shows an example of two segments with different parameters. The choice of each parameter will be investigated in the next chapter.
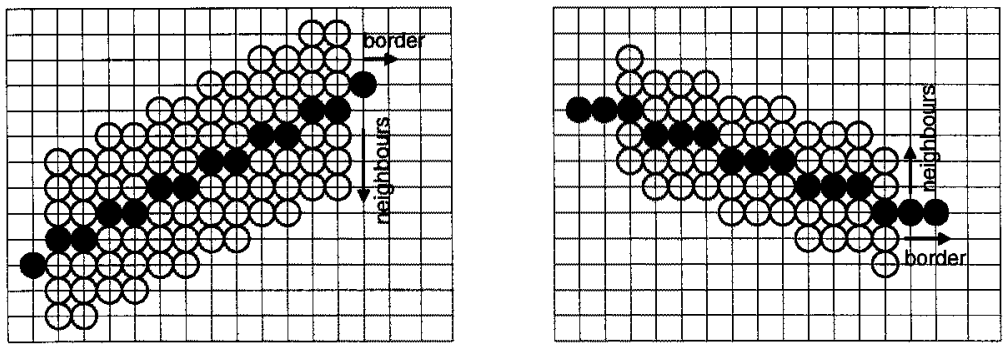

Fig.3. Region parameters: (left) neighbours $=3$, border $=1$, (right) neighbours $=2$, border $=2$ When a fixed set of region parameters is given, the Bresenham-algorithm is very helpful to determine the mean intensity [3]. This algorithm calculates pixel positions only with integer arithmetic and is often used in computer graphics.

\subsection{Experimental Results}

The following analysis is based on five indoor scenes. In order to rate the accuracy of feature estimation subject to different parameters, three criteria are used.

- The standard deviation is calculated taking all pixels in each observed region on each side of the segment into account. The lower the deviation, the more homogenous is the region and the more accurate the mean intensity. The analysis was carried out for a defined percentage of segments with a maximum standard deviation, 
basing on the assumption that in a region with less standard deviation there will be a greater accuracy of the mean intensity estimation.

- The mean gradient over all segments is calculated. The higher the gradient, the more accurate is the estimation, as error pixels shift each mean intensity towards the mean and reduce the distance between both of them, i.e. the gradient.

- Finally a visual measure: the neighbourhood regions of each segment are marked in the original image with the estimated mean intensities.
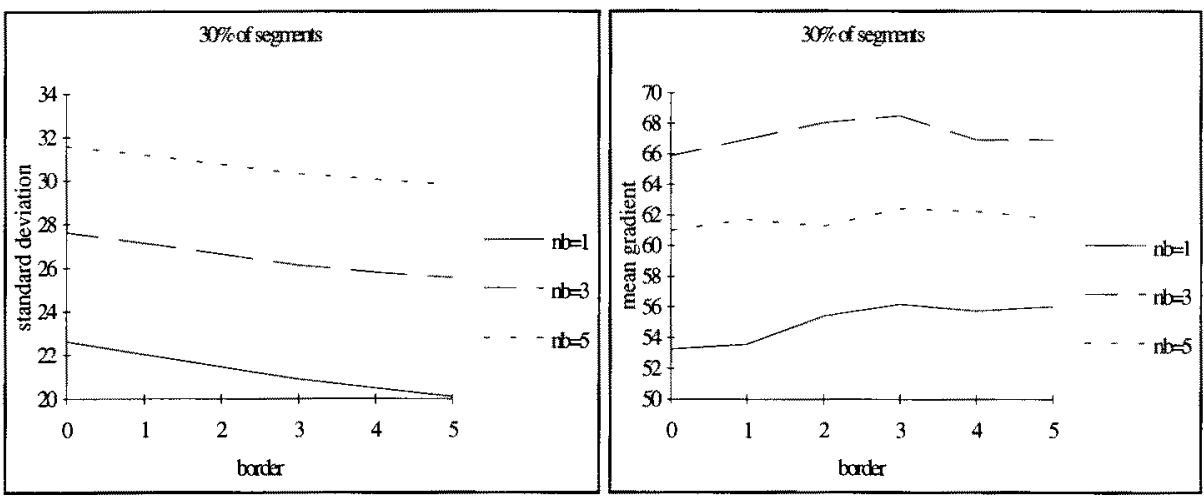

Fig.4. Standard deviation (left) and mean gradient (right) depending on border and neighbourhood (nb)

Regarding Fig.4, the standard deviation decreases with greater border, while the mean gradient increases. This fact is motivated from the segmentation faults at the end of the segment. However, the small change of the mean gradient points to a small influence of the border value.
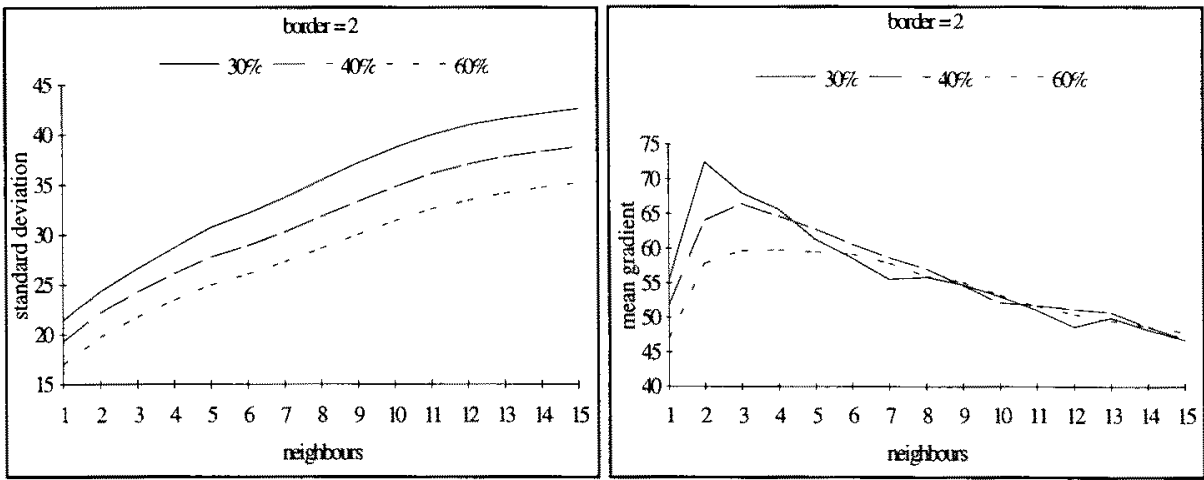

Fig.5. Standard deviation (left) and mean gradient (right) depending on neighbourhood

Fig. 5 shows that, when a fixed border value is given, the standard deviation increases with greater neighbourhood, because the region of true intensity of the edges has been left. It is evident, that the optimal region is where the mean gradient has its maximum, i.e. where the two regions are not influenced by other grey values or neighbouring edges. The analysis, concerning different percentages of considered segments, shows, that segments with high standard deviation need a smaller region than those with lower standard deviation. 

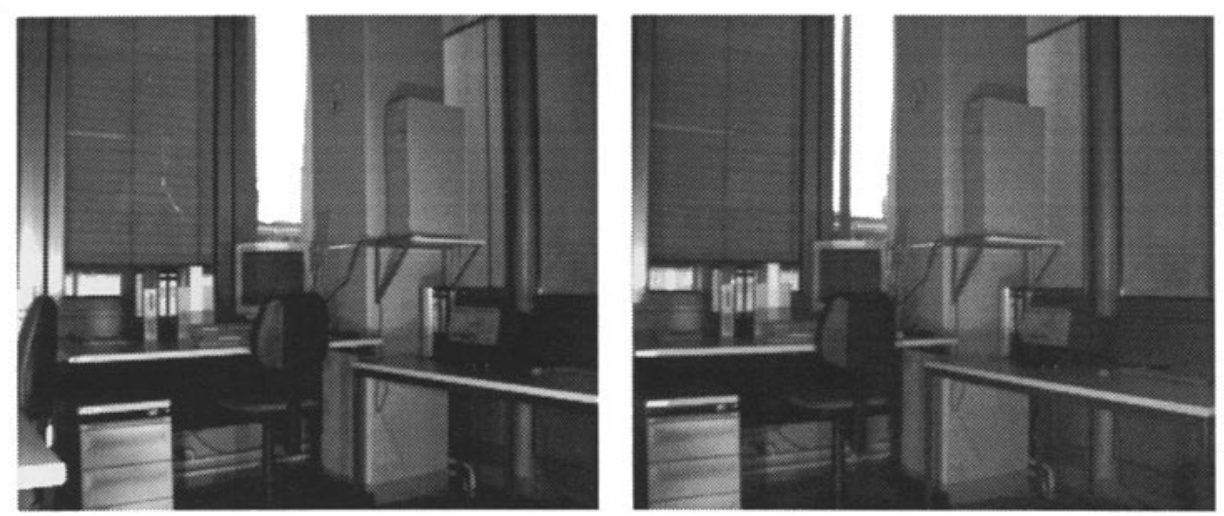

Fig.6. Stereo image pair „scene2“

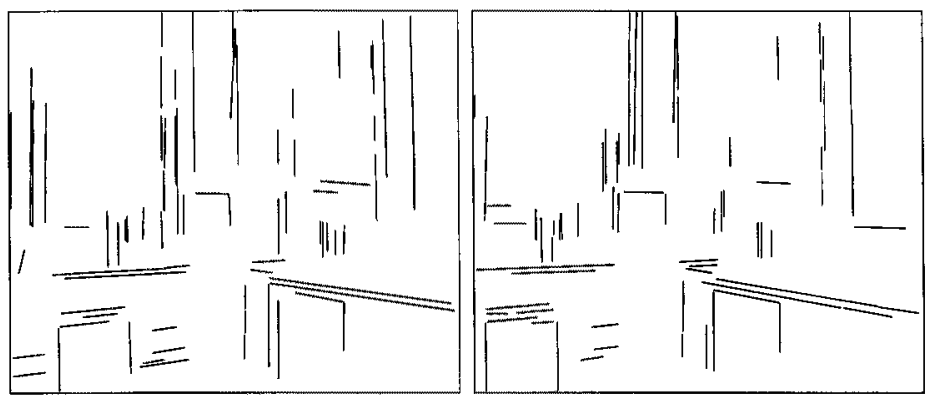

Fig.7. Segment image of "scene2“

The segment image is generated with a sequential segmentation algorithm [9]. The figure below shows a part of the office scene in Fig.6., where the line segments and the region with the estimated mean intensity at each side of the segment are marked.
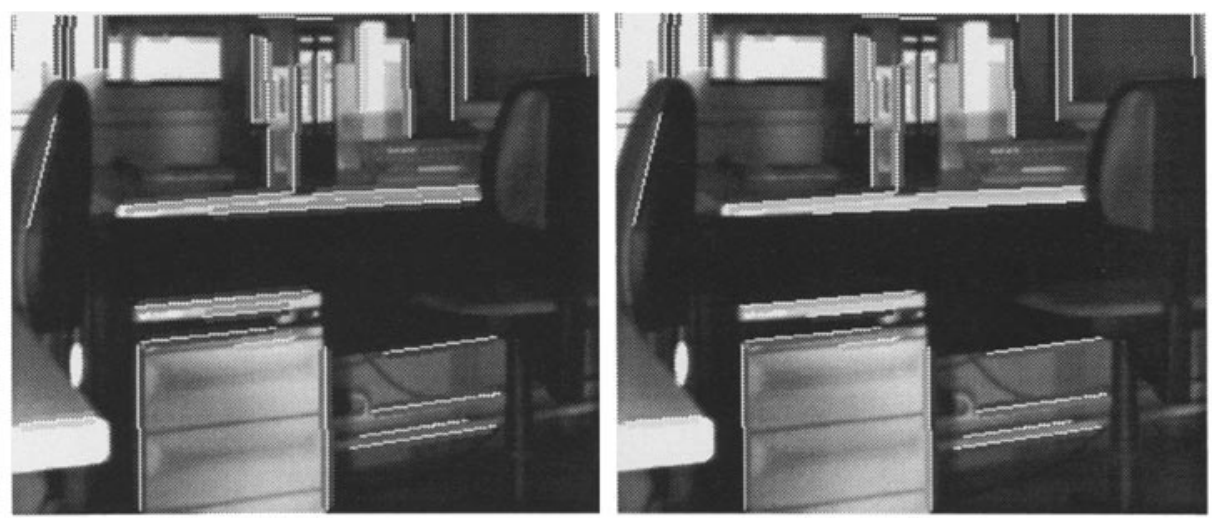

Fig.8. Sequential (left) and global (right) oriented method

In the left image, the sequential-oriented method is applied leading to some errors of the gradient, e.g. at the edges of the drawer. Regarding the global-oriented method, the mean intensities have been estimated with higher accuracy. The computational costs are in the same order. 


\section{Stereo Matching}

The stereo matching algorithm applied in this experiment is based on line segments that are matched according to a three step strategy containing prediction, propagation and validation [1]. This strategy is based on the epipolar and geometrical constraints. In a recursive algorithm different hypotheses will be analysed with respect to the continuity constraint of the disparity for neighbouring segments. This allows a decision whether two segments are possibly homologue, i.e. both segments in the left and right image correspond to the same 3D-edge.

Concerning multiple stereo matching methods in general, it is useful to add further features provided that they meet the following requirements:

- The number of wrong matches tends to decrease, while the number of correct matches increases, which leads to a greater reliability of the matching results.

- The larger computational effort in feature estimation has to be compensated by the subsequent matching algorithm at least.

\subsection{The GLF Constraints}

Defining suitable GLF-constraints, the thresholds have to be determined. A number of stereo image pairs were analysed, and the following characteristics were established:

- The intensity change of homologue segments is always the same. If there is a difference in the sign of gradient, the segments are not homologue and can be excluded, the constraint is binary.

- The mean grey value differs only by 30 grey values between homologue segment pairs. Therefore, it is worthwhile taking into account the different mean values of both images. The constraint is of integer-type.

- The greatest deviation is established at the gradient and differs in some cases by 40 grey level differences. Likewise, this constraint is of integer-type.

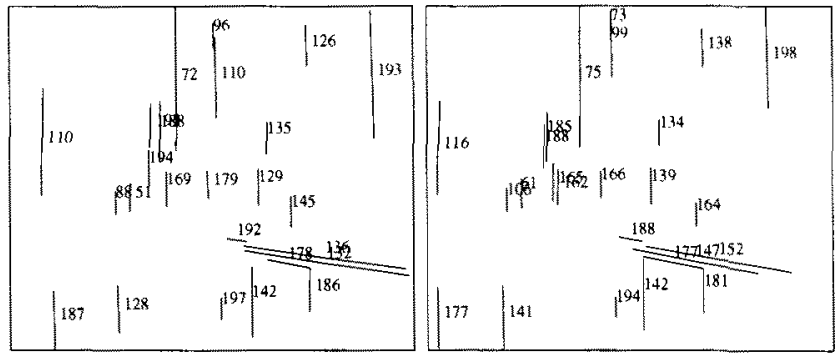

Fig.9. The left and right homologue segments of „scene2“" with registered MGV

\subsection{Matching with GLF}

The segment images, which are to be matched, can be seen in Fig.7. The main characteristics of this image pair are the large number of parallel segments, while only about 65 percent of all segments are part of the same 3D-edge. Fig. 10 shows the corresponding line segments, obtained without using GLF. There are some false matches, marked with corresponding numbers. These segments fulfil the geometrical and epipolar constraint. However, the paralell case could not be resolved. 

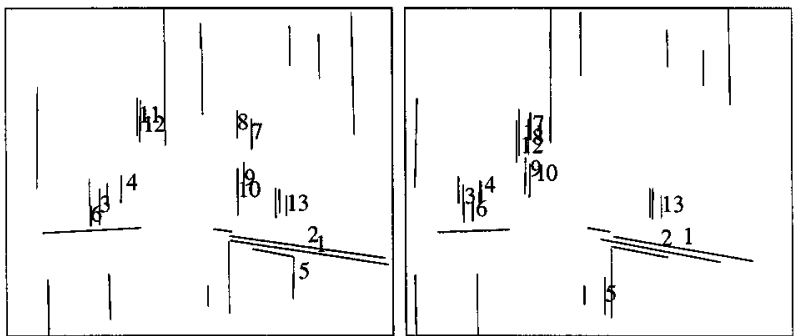

Fig.10. The left and right homologue segments without GLF

The investigation of reliability is based on five indoor scenes with different complexity. The table below shows the results of various features applied. In the first line, the percentage of all matched segments in relation to the total amount of possible matches is registered.

\begin{tabular}{|c||c|c|c|c|c|}
\hline method & without & DIC & DIC+GRAD & DIC+MGV & all GLF's \\
\hline \% of possible matches & 54.3 & 45.57 & 46.42 & 43.93 & 42.34 \\
\hline \% of correct matches & 71.11 & 87.31 & 87.53 & 90.31 & 92.43 \\
\hline
\end{tabular}

Tab. 1. Matching results with different combination of GLF's

If more features are included, the total number of matched segments is lowered, i.e. some of the segments are eliminated. Regarding the second line, it shows that false matches were eliminated, while the percentage of correct matches increases. Considering the factor of computational time, the effort of stereo matching is reduced to about 50 percent, regarding Fig.11. However, there is an additional effort of estimating the GLF, which leads to a total amount which is even lower than without GLF. The effort of estimating the GLF increases in a linear way with the number of segments, while the effort for matching increases exponentially. The use of Direction of Intensity Change (DIC)-feature improves the matching results, but the additional features Gradient (GRAD) and the Mean Grey Value (MGV) are again reducing the computational costs, and the matching is more reliable.

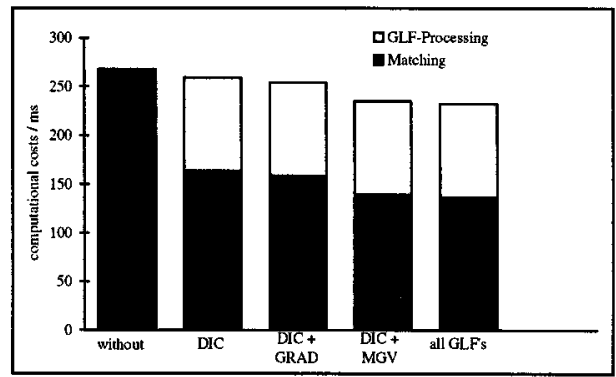

Fig.11. Computational effort with and without GLF

In Fig.12. all three GLF's are applied, observing the following results. The majority of false matches are eliminated and correct match candidates are added. Only three segments have been mismatched in this scene. To conclude, the disambiguity as well as the computational costs have been reduced. 


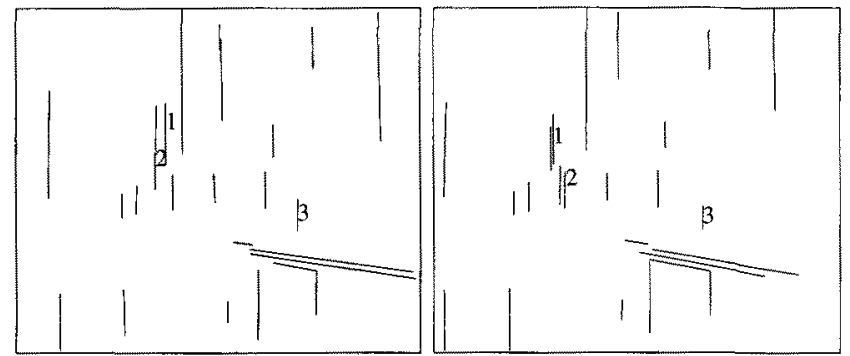

Fig.12. The matching results of "scene 2 to which GLF are applied

\section{Conclusion}

Concerning the estimation of grey-level-features of straight line segments, we described two methods, a sequential-oriented and a global-oriented one. The sequential-oriented method is depending on the edge operator, which estimates the local gradient at each edge point. The global-oriented method can be regarded as the more robust method, using the line segment parameters and providing a fast estimation of the GLF with the Bresenham-algorithm. A statistical analysis and a visual comparison of estimated mean intensity at each side of the edges showed the reliability of the estimated values. In order to investigate the effect of these additional features, they were applied in a line segment based stereo matching algorithm. In general, the additional grey-level features lead to better matching results combined with reduced computational costs. The advantage of this features is the reduction of disambiguity, which primarily occurs in parallel line segments. If the image contains repetitive line structures, these additional features might fail as well. This problem could be solved by a detailed structural analysis of neighbouring line segments.

\section{References}

[1] N. Ayache: „Artificial Vision for Mobile Robots“, MIT Press, Cambridge, Massachusetts, 1991.

[2] O. D. Faugeras: "Three-Dimensional Computer Vision“, MIT Press, Cambridge, Massachusetts, London, England, 1993.

[3] W. D. Fellner: Computer Grafik, Reihe Informatik, Band 58, BI Wissenschaftsverlag. 1988, pp. 95-98.

[4] N. Guil, J. Villalba, E. Zapata: "A Fast Hough Transform for Segment Detection", IEEE Transactions on Image Processing, Vol.4, No.11, Nov. 1995, pp.1541-1548.

[5] R. M. Haralick: "A Facet Model for Image Data, Computer Graphics and Image Processing", Nr. 15, pp. 113-129, from R. Chellappa, A. Sawchuk: Digital Image Processing and Analysis: Vol. 1, IEEE Computer Society Press, Silver Spring 1981.

[6] D. Torkar, N. Pavesic: „Feature Extraction from Aerial Images and Structural Stereo Matching", Int. Conf. on Pattern Recognition, Aug. 1996, Austria.

[7] A. Ude, T. E. Ekre: „Stereo Grouping for Model-Based Recognition“, Int. Conf. on Pattern Recognition, Aug. 1996, Austria.

[8] V. Venkateswar and R. Chellappa: „Hierarchical Stereo Matching Using Feature Groupings", Proc. of Image Understanding Workshop, January 1992.

[9] K. Wall and P. E. Danielson:"A Fast Sequential Method for Polygonal Approximation of Digitized Curves", Pattern Recognition Vol.12, Pergamon Press Ltd., England 1980, pp. 327-331. 